\title{
Coherent-population-trapping resonances with linearly polarized light for all-optical miniature atomic clocks
}

\author{
Sergei A. Zibrov, ${ }^{1,2, *}$ Irina Novikova, ${ }^{3,4, \dagger}$ David F. Phillips, ${ }^{4}$ Ronald L. Walsworth ${ }^{4,5}$ Alexander S. Zibrov, ${ }^{1,2,5}$ \\ Vladimir L. Velichansky, ${ }^{1,2}$ Alexey V. Taichenachev, ${ }^{6,7}$ and Valery I. Yudin ${ }^{6,7,8, \ddagger}$ \\ ${ }^{1}$ P.N. Lebedev Physical Institute RAS, RU-117924 Moscow, Russia \\ ${ }^{2}$ Moscow State Engineering Physics Institute, RU-115409 Moscow, Russia \\ ${ }^{3}$ Department of Physics, College of William \& Mary, Williamsburg, Virginia 23185, USA \\ ${ }^{4}$ Harvard-Smithsonian Center for Astrophysics, Cambridge, Massachusetts, 02138, USA \\ ${ }^{5}$ Department of Physics, Harvard University, Cambridge, Massachusetts, 02138, USA \\ ${ }^{6}$ Institute of Laser Physics SB RAS, Novosibirsk RU-630090, Russia \\ ${ }^{7}$ Novosibirsk State Technical University, Novosibirsk RU-630092, Russia \\ ${ }^{8}$ Novosibirsk State University, Novosibirsk RU-630090, Russia \\ (Received 25 October 2009; published 28 January 2010)
}

\begin{abstract}
We present a joint theoretical and experimental characterization of the coherent population trapping (CPT) resonance excited on the $D_{1}$ line of ${ }^{87} \mathrm{Rb}$ atoms by bichromatic linearly polarized laser light. We observe high-contrast transmission resonances (up to $\approx 25 \%$ ), which makes this excitation scheme promising for miniature all-optical atomic clock applications. We also demonstrate cancellation of the first-order light shift by proper choice of the frequencies and relative intensities of the two laser-field components. Our theoretical predictions are in good agreement with the experimental results.
\end{abstract}

DOI: 10.1103/PhysRevA.81.013833

PACS number(s): 42.72.-g, 42.50.Gy, 32.70.Jz

\section{INTRODUCTION}

There is great interest in the development of miniature (chip scale) atomic clocks, magnetometers, and other metrology tools. One of the promising approaches utilizes coherent population trapping (CPT) resonances: By coherent interaction with two resonant optical fields in a $\Lambda$ configuration, atoms can be prepared in a noninteracting quantum superposition of two hyperfine ground states (a "dark" state), which results in enhanced transmission [1]. The dark state is very sensitive to detuning of the frequency difference of the two optical fields from the hyperfine splitting of the atomic states, which allows using the CPT effect for precision sensing. In recent years, atomic frequency standards based on CPT resonances with fractional frequency stability of $10^{-12}$ or better were demonstrated [1-3]. Further improvements in stability require realization of CPT resonances with optimized parameters, such as larger amplitude and contrast and smaller linewidth. In addition, any sensitivities to environmental changes must be minimized. In particular, light shifts (i.e., spectra shifts in the transmission peak with total light power) can limit the frequency stability of CPT-based devices.

Miniature atomic clocks typically use a single diode laser (usually a vertical cavity surface-emitting laser, or VCSEL) phase modulated at an appropriate frequency to obtain two light fields, required for CPT resonances. As a result, practical operational schemes are restricted to light fields of the same polarization. In the case of circularly polarized light fields, for example, this restriction leads to a significant reduction of CPT resonance amplitude since a large fraction of the atomic population becomes trapped in the "end" Zeeman states with

\footnotetext{
*szibrov@yandex.ru

†inovikova@physics.wm.edu

†viyudin@mail.ru
}

the maximum value of the angular momentum $\left(m_{F}=F\right.$ or $\left.m_{F}=-F\right)$ and not in the desired magnetic-field-insensitive states with $m_{F}=0$ [4]. To mitigate this problem, a variety of CPT schemes [5-8] as well as related coherent resonances [9-11] were proposed and studied by different groups.

In this article we present a CPT interaction scheme that takes advantage of the unique level combination of alkali-metal atoms with nuclear spin $I=3 / 2$ to realize high-contrast magneto-insensitive CPT resonances with two optical fields of the same linear polarization (lin||lin configuration). This scheme, initially proposed in Ref. [12], is possible when two ground states with total angular momentum $F_{g}=1,2$ are coupled only through an excited state with total angular momentum $F_{e}=1$. In a vapor cell this situation is realized only for the $D_{1}$ line of ${ }^{87} \mathrm{Rb}$ when the exited-state hyperfine levels are spectrally resolved. Here, we present an experimental and theoretical study of this lin||lin CPT scheme and demonstrate the cancellation of the first-order light shift by proper choice of the frequencies and relative intensities of the two optical fields, which can reduce the sensitivity of resonance parameters to laser intensity fluctuations.

\section{THEORETICAL ANALYSIS}

The observation of CPT resonances requires two optical fields $\mathbf{E}_{1,2} e^{\left[-i\left(\omega_{1,2} t-k_{1,2} z\right)\right]}$ tuned to two-photon resonance with the hyperfine transition of an appropriate atom $\omega_{1}-\omega_{2}=$ $\Delta_{\text {hfs }}$. In ${ }^{87} \mathrm{Rb}$, for example, the hyperfine splitting is $\Delta_{\mathrm{hfs}} \simeq$ $6.835 \mathrm{GHz}$. For miniature atomic clock applications, both fields are produced by a single phase-modulated laser (typically a VCSEL) and have the same polarization. Traditional CPT clocks use circularly polarized laser output [1]. In this case the magnetic-field-insensitive CPT resonance is formed between $m_{F}=0$ Zeeman sublevels [(0)-(0) clock transition]. For example, for $\sigma_{+}$-polarized laser fields $E_{+}^{(1,2)}$, 
the corresponding CPT dark state is

$$
\begin{aligned}
& \left|\operatorname{dark}_{\text {circ }}\right\rangle \\
& \quad=\mathcal{N}\left[\left|F_{g}=1, m=0\right\rangle-\frac{V_{0+}^{(1)} E_{+}^{(1)}}{V_{0+}^{(2)} E_{+}^{(2)}}\left|F_{g}=2, m=0\right\rangle\right],
\end{aligned}
$$

where $V_{0}^{(1,2)}$ are the transition matrix elements for $\mid F_{g}=$ $1,2, m=0\rangle \rightarrow\left|F_{e}, m=1\right\rangle$ and $\mathcal{N}$ is a normalization coefficient. The disadvantage of such a scheme is the existence of the noninteracting trap state $\mid$ trap $\left._{\text {circ }}\right\rangle=\left|F_{g}=2, m=2\right\rangle$ : Since it is decoupled from both circularly polarized optical fields, a large portion of the atomic population is optically pumped in the trap state. This effectively reduces the density of interacting atoms, resulting in a lower CPT resonance contrast.

The application of linearly (or in general elliptically) polarized optical fields can eliminate such trap states. However, such polarizations simultaneously deteriorate the ground-state coherence formed between the two magnetic-field-insensitive sublevels $m=0$ in the presence of a longitudinal magnetic field. In this case, two opposite circularly polarized components of the optical fields simultaneously form two $\Lambda$ systems between the $m=0$ ground states, through both $\left|F_{e}, m= \pm 1\right\rangle$ excited states. The matrix elements for $\left|F_{g}=2, m=0\right\rangle \rightarrow$ $\left|F_{e}, m= \pm 1\right\rangle$ are equal and opposite $V_{0+}^{(2)}=-V_{0-}^{(2)}$, but those for the transitions $\left|F_{g}=1, m=0\right\rangle \rightarrow\left|F_{e}, m= \pm 1\right\rangle$ are the same $V_{0+}^{(1)}=V_{0-}^{(1)}$. As a result, the dark states for each $\Lambda$ system are different, as can be seen from Eq. (1). In particular, for linearly polarized light, when the intensities of the two circular components are the same, the ground-state coherence on the (0)-(0) clock transition disappears completely since the dark state for one $\Lambda$ system becomes the bright (interacting) state for the other.

${ }^{87} \mathrm{Rb}$ atoms, with the nuclear spin of $I=3 / 2$, offer a unique possibility to form high-contrast magneto-insensitive CPT dark resonances using a different combination of Zeeman sublevels with arbitrary polarized light fields even for the condition of reduced or zero coherence on the (0)-(0) clock transition. Possible $D_{1}$ optical transitions from the $F_{g}=1,2$ ground state to the $F_{e}=1$ excited state resulting from the interaction of two elliptically polarized optical fields are shown in Fig. 1. The solid arrows show two possible twophoton $\Lambda$ resonances that create atomic coherence between

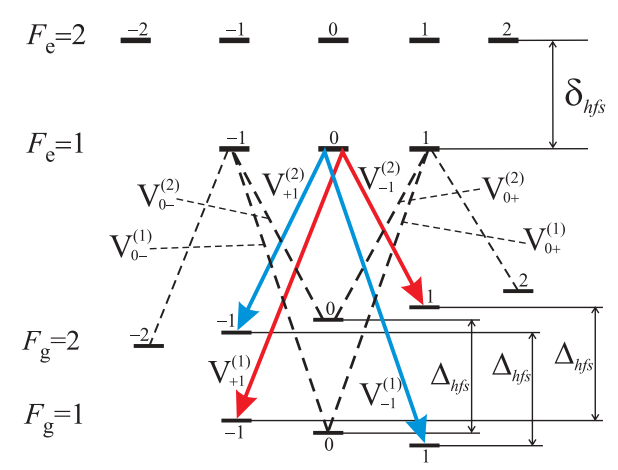

FIG. 1. (Color online) Light-induced two-photon transitions in a bichromatic field from $F_{g}=1,2$ to $F_{e}=1$ for different variants of optical polarization.
$\left|F_{g}=1, m=-1\right\rangle,\left|F_{g}=2, m=+1\right\rangle$ and between $\mid F_{g}=1$, $m=+1\rangle,\left|F_{g}=2, m=-1\right\rangle$ [further in the text we refer to these as $(-1)-(+1)$ and $(+1)-(-1)$ resonances]. Both of these $\Lambda$ schemes are excited through a common excited state $\left|F_{e}=1, m=0\right\rangle$. Most importantly, the gyromagnetic $g$-factor of the two ground-state hyperfine levels in $I=3 / 2$ alkali-metal atoms have equal absolute value and opposite signs (neglecting the small contribution of nuclear spin). Thus both ground states in each of these $\Lambda$ systems shift by an equal amount when a weak-longitudinal magnetic field is applied. As a result a CPT resonance occurs at the unshifted "clock" frequency $\omega_{1}-\omega_{2}=\Delta_{\mathrm{hfs}}$ via two dark states $\left|\operatorname{dark}_{ \pm}\right\rangle$

$$
\begin{aligned}
& \left|\operatorname{dark}_{ \pm}\right\rangle \\
& \quad=\mathcal{N}_{ \pm}\left[\left|F_{g}=1, m=\mp 1\right\rangle-\frac{V_{ \pm 1}^{(1)} E_{ \pm 1}^{(1)}}{V_{\mp 1}^{(2)} E_{\mp 1}^{(2)}}\left|F_{g}=2, m= \pm 1\right\rangle\right] .
\end{aligned}
$$

Here $V_{ \pm 1}^{(1,2)}$ are the transition matrix elements shown in Fig. 1 and $\mathcal{N}_{ \pm}$are the normalization constants. In this configuration there are no "trap" states when the Zeeman levels are not degenerate. Hence, under CPT conditions the atomic population accumulates only in the coherent dark states, resulting in higher CPT resonance contrast.

While the states in Eq. (2) exist for arbitrary elliptical polarizations of the optical fields, a linearly polarized laser output is preferable since the influence of nuclear spin leads to a small difference in the $g$-factor for different hyperfine components of the ground state. As a result of this $g$-factor difference the frequencies of the $(-1)-(+1)$ and $(+1)-(-1)$ two-photon $\Lambda$ resonances are also different in the finite magnetic field (for ${ }^{87} \mathrm{Rb}$ this difference is $2.8 \mathrm{kHz} / \mathrm{G}$ ), but their position is symmetric relative to the hyperfine splitting frequency $\Delta_{\mathrm{hfs}}$. Thus, for the lin||lin excitation scheme, this small difference in the two-photon resonance frequencies leads only to the broadening of the resonance, but not to a frequency shift (in the linear Zeeman effect approximation). At a stronger magnetic field the CPT resonances from the two $\Lambda$ systems become resolvable; the dip between the two CPT peak may be used as a "pseudo-resonance" for atomic clocks as well, although its characteristics look less promising [13,14].

It is important to note that if the ground states $\mid F_{g}=$ $1, m= \pm 1\rangle$ and $\left|F_{g}=2, m= \pm 1\right\rangle$ are coupled to the excited states $\left|F_{e}=2, m= \pm 2\right\rangle$, then the dark state given by Eq. (2) is degraded [12,15]. Thus, the key requirement to the observation of high-contrast $\operatorname{lin}||$ lin $\mathrm{CPT}$ resonances is a good spectral resolution of the excited state $F_{e}=1$. The $D_{1}$ line of ${ }^{87} \mathrm{Rb}$ is the most promising candidate for this scheme since its excited-state hyperfine splitting $(812 \mathrm{MHz})$ exceeds the Doppler broadening at room temperature, allowing its use in vapor cells and hence in miniature atomic clocks. For all other $I=3 / 2$ alkali-metal atoms $\left({ }^{7} \mathrm{Li},{ }^{23} \mathrm{Na}\right.$, and ${ }^{39,41} \mathrm{~K}$ ) and also for the $D_{2}$ line of ${ }^{87} \mathrm{Rb}$, a good spectral resolution of the excited state is possible only in the case of laser cooled atoms or in a collimated atomic beam. Even for the ${ }^{87} \mathrm{Rb} D_{1}$ line, the realization of miniature lin||lin CPT atomic clocks will require careful optimization of the buffer gas pressure to increase the diffusion time of atoms in the laser beam and hence the dark-state lifetime without severe 


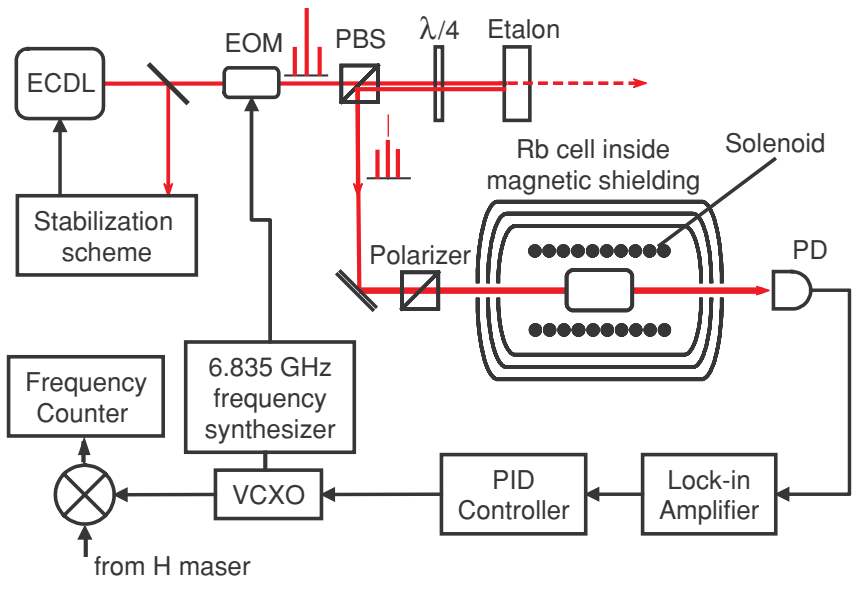

FIG. 2. (Color online) Schematic of the experimental setup. See text for abbreviations.

degradation of the resonance amplitude due to the reduced spectral resolution of the pressure-broadened excited states. In particular, there is a potential difficulty in using this scheme for chip-scale atomic clocks that typically use very high buffer gas pressures, which broaden the optical resonances beyond the usual Doppler linewidth. This difficulty can be circumvented, however, through the addition of an antirelaxation wall coating to vapor cells with lower buffer gas pressure [16].

\section{EXPERIMENTAL PROCEDURE}

A schematic of the experimental setup is shown in Fig. 2. To produce the required resonant bichromatic field the output of an external cavity diode laser (ECDL) was externally phase modulated at the ${ }^{87} \mathrm{Rb}$ hyperfine frequency $6.835 \mathrm{GHz}$ by an electro-optical modulator (EOM). The laser frequency was stabilized to a saturation spectroscopy resonance in a vacuum reference cell, with the drive field (unmodulated carrier) tuned in the vicinity of the $5 S_{1 / 2} F_{g}=2 \leftrightarrow 5 P_{1 / 2} F_{e}=1$ transition and the probe field (first high-frequency modulation sideband) resonant with the $5 S_{1 / 2} F_{g}=1 \leftrightarrow 5 P_{1 / 2} F_{e}=1$ transition. The two-photon detuning between the probe and drive fields was precisely controlled by varying the output frequency of the microwave synthesizer providing the EOM modulation signal. The intensity ratio $I_{1} / I_{2}$ between both the probe and drive fields (sideband-to-carrier ratio) was adjusted up to $40 \%$ by changing the power of the microwave signal sent to the EOM. To achieve higher intensity ratios we used a thermostabilized quartz Fabry-Perot etalon with free spectral range of $20 \mathrm{GHz}$, finesse of 30, and maximum transmission of $70 \%$, temperaturetuned to partially transmit the strong drive field. The beam reflected from the etalon contained the unaffected first-order sidebands and an attenuated drive field. The attenuation was controlled by fine-tuning the transmission resonance of the etalon via temperature. The reflected beam was collimated to a diameter of $7 \mathrm{~mm}$ and linearly polarized by a quartz polarizer before entering the $\mathrm{Rb}$ vapor cell.

All experimental measurements were recorded using a cylindrical Pyrex cell (diameter $=2.5 \mathrm{~cm}$, length $=4 \mathrm{~cm}$ ) containing isotopically enriched ${ }^{87} \mathrm{Rb}$ and 4 Torr of $\mathrm{Ne}$ buffer gas to increase the $\mathrm{Rb}$ interaction time with the laser beam. The cell was mounted inside three layers of magnetic shielding and maintained at a constant temperature of $40^{\circ} \mathrm{C}$. To separate the magneto-insensitive "clock" resonances $\left(\mid F_{g}=\right.$ $1, m=-1\rangle \leftrightarrow\left|F_{g}=2, m=+1\right\rangle$ and $\left|F_{g}=1, m=+1\right\rangle \leftrightarrow$ $\left.\left|F_{g}=2, m=-1\right\rangle\right)$ from the field-dependent resonances, a uniform longitudinal magnetic field of approximately 180 $\mathrm{mG}$ was applied using a solenoid mounted inside the innermost magnetic shield. A photodetector (PD) placed after the cell was used to measure the transmitted optical signal. CPT resonance parameters such as linewidth, amplitude, and so on were determined for each set of experimental conditions.

To continually monitor the CPT resonance frequency the EOM modulation frequency was locked to the CPT resonance using phase-sensitive detection. A slow frequency modulation at $f_{m}=400 \mathrm{~Hz}$ was superimposed on the $6.835 \mathrm{GHz}$ microwave synthesizer output; then the PD signal was demodulated at $f_{m}$ using a lock-in amplifier. The resulting error signal was fed back to lock the frequency of the voltage controlled crystal oscillator (VCXO) that controlled the synthesizer. The frequency of the locked VCXO was measured by beating it with a stable reference derived from a hydrogen maser.

\section{EXPERIMENTAL RESULTS}

Figure 3(a) shows typical measured CPT resonances in the lin||lin configuration. The strong peak at zero twophoton detuning is from the magneto-insensitive $(-1)-(+1)$ and $(+1)-(-1)$ CPT resonances; the two smaller side peaks correspond do the magnetic-field-sensitive resonances
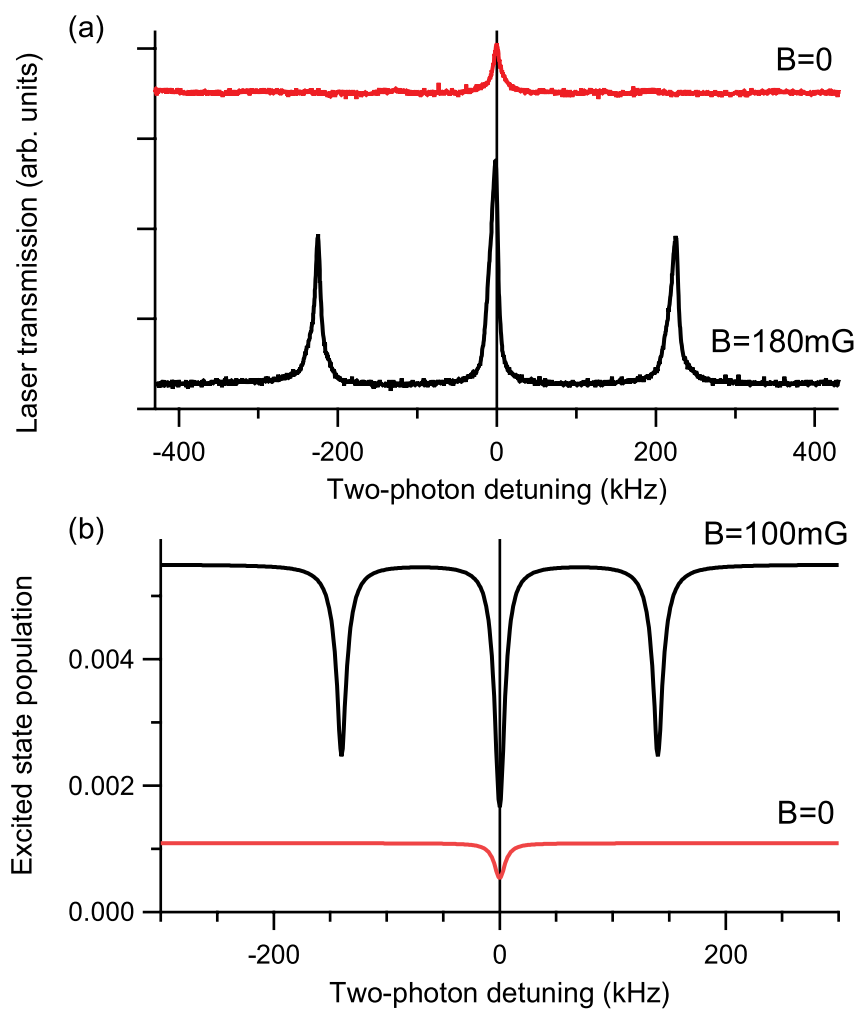

FIG. 3. (Color online) (a) Examples of measured CPT resonances at $B=0$ and $B=180 \mathrm{mG}$. Laser intensity before the cell is $8.4 \mathrm{~mW} / \mathrm{cm}^{2}$. (b) Calculated excited-state population $\pi_{e}$ versus two-photon detuning at $B=0$ and $B=100 \mathrm{mG}$. Total resonant intensity $I_{1}+I_{2}=10 \mathrm{~mW} / \mathrm{cm}^{2}$. 

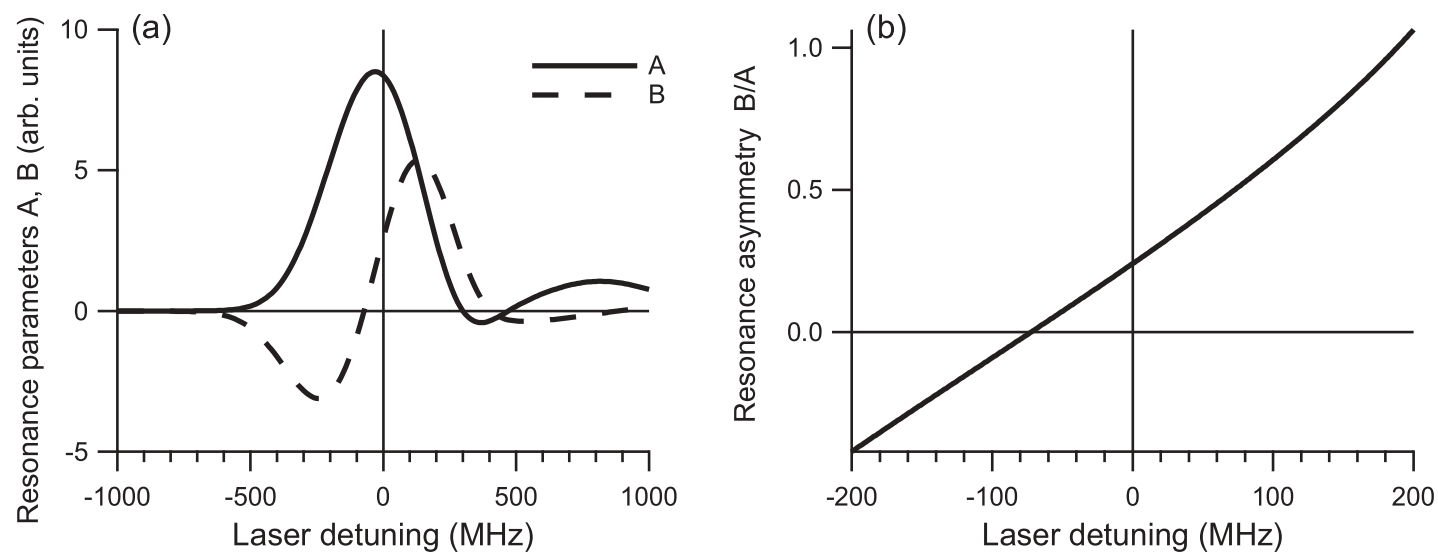

FIG. 4. (a) Calculated values of the line shape parameters $A$ and $B$ [Eq. (3)] that describe the symmetric and antisymmetric components of a CPT resonance as a function of laser detuning $\delta_{L}$ from the $F_{e}=1$ level. In these calculations sideband-to-carrier ratio $I_{1} / I_{2}=0.5$; homogeneous collisionally broadened optical linewidth $\gamma=20 \mathrm{MHz}$; ground-state relaxation rate $\Gamma=500 \mathrm{~Hz}$, and the laser beam diameter $6.9 \mathrm{~cm}$. (b) Calculated CPT resonance asymmetry $B / A$ as a function of laser detuning $\delta_{L}$ for $I_{1} / I_{2}=0.5, \gamma=20 \mathrm{MHz}, \Gamma=500 \mathrm{~Hz}$, and beam diameter $6.9 \mathrm{~cm}$. CPT resonance becomes symmetric $(B=0)$ at $\delta_{L}=-72 \mathrm{MHz}$.

$(+1)-(+1)$ and $(-1)-(-1)$ formed between the $\mid F_{g}=2, m=$ $\pm 1\rangle$ and $\left|F_{g}=1, m= \pm 1\right\rangle$. Note that when the Zeeman levels are degenerate (at $B=0$ ), coupling to the trap states $\left|F_{g}=2, m= \pm 2\right\rangle$ significantly reduces the amplitude and contrast of the CPT resonance (top line). A small magnetic field eliminates coupling to these trap states, resulting in much higher resonance contrast. Figure 3(b) shows the calculated dependence of the total excited state populations $\pi_{e}$ as a function of two-photon detuning. These calculated spectra can be directly compared to the measured CPT resonances since $\pi_{e}$ is proportional to the laser field absorption [17]; small discrepancies arise from residual magnetic field gradients.

We modeled each CPT-resonance line shape as a function of the two-photon (Raman) detuning $\delta_{R}$ by a generalized Lorentzian [17,18]

$$
I\left(\delta_{R}\right)=I_{b g}+\frac{A(\tilde{\gamma} / 2)^{2}}{(\tilde{\gamma} / 2)^{2}+\left(\delta_{R}-\delta_{0}\right)^{2}}+\frac{B\left(\delta_{R}-\delta_{0}\right) \tilde{\gamma} / 2}{(\tilde{\gamma} / 2)^{2}+\left(\delta_{R}-\delta_{0}\right)^{2}},
$$

where $\tilde{\gamma}$ is the width of the CPT resonance, $\delta_{0}$ is the resonance shift, $A$ and $B$ characterize the amplitudes of the symmetric and antisymmetric Lorentzian components, and $I_{b g}$ is the total transmission away from CPT resonance (background transmission). See the Appendix for details. $A, B, \tilde{\gamma}$, and $\delta_{0}$ are functions of the optical field amplitudes and one-photon detuning $\delta_{L}$; their values were extracted by fitting experimentally recorded or numerically calculated CPT resonances to Eq. (3). Figure 4(a) shows the calculated variation of $A$ and $B$ when the laser frequency is swept across the optical transition $F_{g}=2 \rightarrow F_{e}=1$ for typical experimental conditions: One sees that the symmetric Loretzian component $A$ reaches its maximum near the optical resonance; whereas the antisymmetric component $B$ is largest for $\delta_{L} \approx \pm 200 \mathrm{MHz}$. This resonance asymmetry can lead to shifts in measured resonance position if a phase-sensitive-detection method is used [19]. Thus, it is desirable to operate a CPT atomic clock under conditions that lead to a symmetric CPT resonance. Figure 4(b) shows that for the lin||lin configuration, CPT resonances become symmetric $(B=0)$ when the laser frequency is appropriately red detuned from the $F_{e}=1$ excited state. We found a similar value for the optimal detuning experimentally. Note that this detuning also corresponds to the maximum of the the amplitude for the symmetric component $A$, making these operational conditions attractive for clock operation.

Figure 5(a) shows the measured dependance of the CPT resonance width as a function of total laser power, for the optimal laser detuning $\left(\delta_{L}=-72 \mathrm{MHz}\right)$ to achieve a symmetric resonance. The results of numerical calculations (solid lines) follow the experimental data quite closely; both
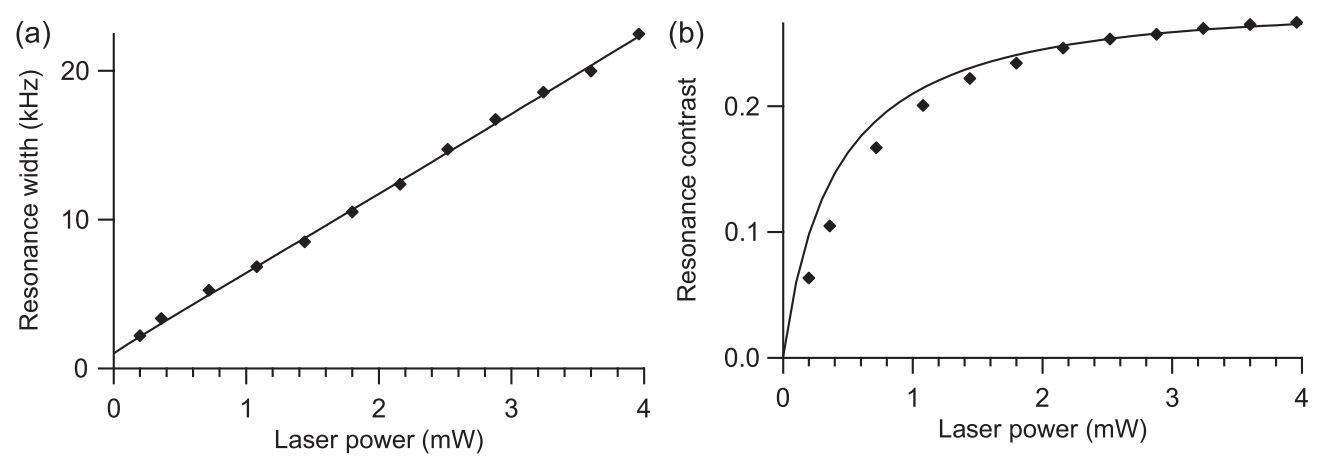

FIG. 5. (a) lin||lin CPT resonance linewidth $\tilde{\gamma}$ and (b) the resonance contrast $C$ (amplitude to background ratio), for optimal laser detuning to achieve a symmetric resonance. Experimental data points are shown as diamonds; solid lines represent numerical calculations. 
show the expected linear power broadening. Figure 5(b) shows experimental data and numerical calculations for the resonance contrast, defined as the ration between the resonance amplitude and the off-resonant background transmission $C=A / I_{b g}$. The contrast increases rapidly with the laser power and then saturates at $C>25 \%$ for total incident laser power $\geqslant 1 \mathrm{~mW}$. This maximum contrast greatly exceeds that achieved to date with traditional CPT clock resonances, $C<4 \%$ [1], albeit with relatively large laser power and resonance width $\sim 10 \mathrm{kHz}$.

The fractional frequency stability of a microwave oscillator locked to the CPT resonance is proportional to the quality figure $q=C / \widetilde{\gamma}$-the ratio between the resonance contrast and width. Notably, $q$ characterizes the achievable stability for both shot-noise-limited and intensity-fluctuation-limited atomic clocks [20]. If the frequency stability of an atomic clock is limited by photon shot noise, the expected Allan deviation, $\sigma(\tau)$ is given by [21]

$$
\sigma(\tau)=\frac{1}{4} \sqrt{\frac{\eta e}{I_{b g}}} \frac{1}{q \nu_{0}} \tau^{-1 / 2},
$$

where $v_{0}=\Delta_{\mathrm{hfs}}$ is the atomic reference frequency, $e$ is the electron charge, $\eta$ is the PD sensitivity (measured optical energy per photoelectron), and $\tau$ is the integration time. For the lin||lin CPT resonances in our experiment (optimal laser detuning), the maximum $q \approx 3.2 \times 10^{-5} / \mathrm{Hz}$ value was measured at $\approx 800 \mu \mathrm{W}$ of laser power, which corresponds to a resonance contrast $C \simeq 17 \%$ and width $\tilde{\gamma} \simeq 5 \mathrm{kHz}$. This value implies a shot-noise-limited frequency stability of $\sigma(\tau) \sim 2 \times 10^{-14} \tau^{1 / 2}$. In practice, however, the frequency stability will also be limited by residual intensity noise that depends strongly on the linewidth of the laser [22,23]. Recently, a table-top prototype atomic clock based on lin||lin CPT resonances with a short-term fractional frequency stability $2 \times 10^{-11} \tau^{-1 / 2}$ was demonstrated [24].

\section{CANCELLATION OF FIRST-ORDER LIGHT SHIFT}

The light shift of a CPT resonance (i.e., changes in the maximum transmission caused by changing laser intensity [25]) is another important characterization parameter for clock

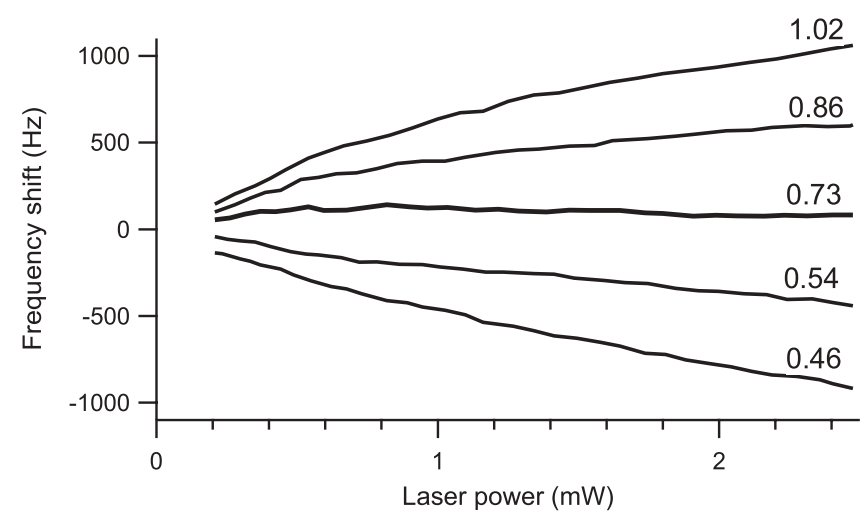

FIG. 6. Measured CPT resonance frequency shift as a function of laser power for different sideband-to-carrier ratios (shown for each trace). Experimental conditions were otherwise the same as for Fig. 5. Ratio of 0.73 corresponds to the light shift compensation condition (i.e., CPT resonance frequency minimally affected by laser power variation).

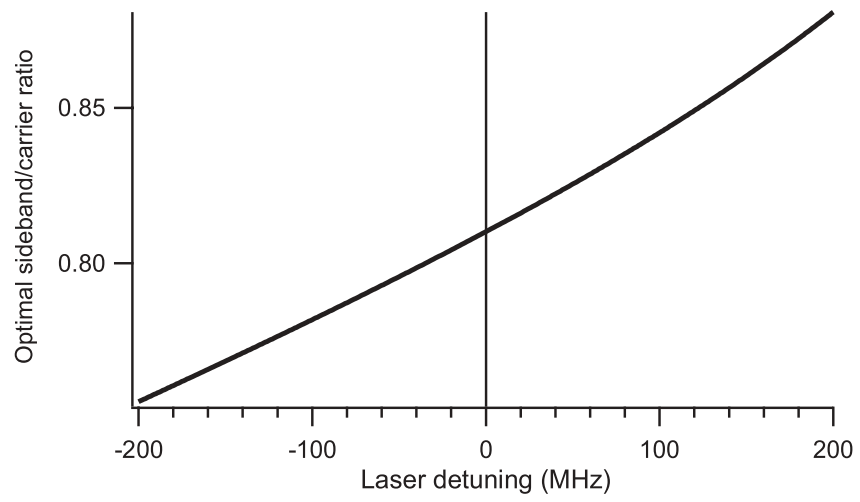

FIG. 7. Calculated optimal sideband-to-carrier ratio for which light shift of CPT resonance position is minimally dependent on laser power, as a function of laser detuning.

applications. Light shifts deteriorate the stability of clocks since they directly transfer any instabilities in the laser intensity into variation of the measured clock frequency. In the lin||lin configuration the sensitivity of the CPT resonance position to laser intensity may be strongly reduced by choosing a proper intensity ratio between two optical fields. For a resonant $\Lambda$ system, the main contribution to the CPT resonance light shift comes from the coupling to nonresonant hyperfine levels, and in particular, to the upper hyperfine level $F_{e}=2$ since $\delta_{\text {hfs }}=812 \mathrm{MHz} \ll \Delta_{\mathrm{hfs}}=6.835 \mathrm{GHz}$. To motivate first-order light shift cancellation we consider the two limiting cases when one of the optical fields is much stronger than the other. If $\left|E_{1} / E_{2}\right| \gg 1$, the dark resonance position is mostly affected by the light shift of the Zeeman sublevel $\left|F_{g}=1, m= \pm 1\right\rangle$ caused by the strong $E_{1}$ field. This Zeeman shift is negative and proportional to $-\left|V^{(1)} E_{1}\right|^{2} / \delta_{\mathrm{hfs}}$ and results in a positive shift of the CPT resonance. In the opposite case $\left|E_{1} / E_{2}\right| \ll 1$, the main contribution is due to the shift of the Zeeman sublevel $\left|F_{g}=2, m= \pm 1\right\rangle \propto-\left|V^{(2)} E_{2}\right| / \delta_{\text {hfs }}$, which, in this case, leads to a negative shift of the CPT resonance. Since, in general, the frequency of the CPT peak will be determined by light shifts created by both fields, it is reasonable to expect that by changing the sideband-to-carrier ratio we can find a point of light shift cancellation. Our calculations show that the compensation condition depends only on the proper choice of the ratio of the two fields and not on the absolute value of either optical component. For the optimal one-photon laser detuning $\delta_{L}=-72 \mathrm{MHz}$, the calculations predict $I_{1} / I_{2}=0.79$ for light shift compensation, which is in good agreement with the experimentally determined $I_{1} / I_{2}=0.73$ (see Fig. 6). The compensation ratio depends slightly on laser detuning, as is shown in Fig. 7.

\section{CONCLUSION}

In this article we present both theoretical and experimental characterizations of magnetic field-insensitive CPT resonances induced in ${ }^{87} \mathrm{Rb}$ vapor by linearly polarized light. This excitation scheme allows us to greatly increase the contrast of magneto-insensitive CPT resonances. The dependance of the resonance amplitude, linewidth, contrast, quality figure, and frequency on total laser intensity is investigated. We also demonstrate light-shift compensation by proper choice of the 
frequencies and intensity ratio of the resonant optical fields. Our results suggest that lin||lin CPT resonances may be good candidates for miniature atomic clocks.

\section{ACKNOWLEDGMENTS}

The authors are grateful to Eugeniy E. Mikhailov for useful discussions. This work was supported by the Smithsonian Institution. I.N. acknowledges support from Jeffress Research Grant No. J-847. A.V.T and V.I.Y. were supported by RFBR (Grant Nos. 08-02-01108, 08-07-00127, 09-02-01151, 10-0200406, and 10-08-00844), the Russian Academy of Science, Presidium SB RAS, and by the federal programs "Development of scientific potential of higher school 2009-2010" and "Scientific and pedagogic personnel of innovative Russia 2009-2013."

\section{APPENDIX: DENSITY MATRIX CALCULATIONS}

Our numerical calculations employ a model based on the standard density-matrix approach (see, e.g., Ref. [17]). Under assumptions of low saturation and total collisional depolarization of the excited state, the ground-state density matrix $\widehat{\sigma}_{g g}$ obeys the following equations

$$
\begin{aligned}
\frac{d}{d t} \widehat{\sigma}_{g g} & =-i\left[\widehat{H}_{\mathrm{eff}} \widehat{\sigma}_{g g}-\widehat{\sigma}_{g g} \widehat{H}_{\mathrm{eff}}^{\dagger}\right]+\left(\frac{\pi_{e}}{\tau_{e}}+\Gamma\right) \frac{\widehat{\Pi}_{g}}{n_{g}}, \\
\operatorname{Tr}\left\{\widehat{\sigma}_{g g}\right\} & =1,
\end{aligned}
$$

where $\widehat{\Pi}_{g}$ is the unity matrix in the ground-state manifold, $n_{g}=2(2 I+1)$ is the total number of substates in the ground state, and $\pi_{e}$ is the total population of the excited state. The first source term in Eq. (A1), inversely proportional to the excitedstate radiative lifetime $\tau_{e}$, describes isotropic repopulation of the ground-state sublevels due to spontaneous decay of the excited states. The second source term, proportional to the ground-state relaxation rate $\Gamma$, describes the entrance of unpolarized atoms into the laser beam due to diffusion and collisions. Hereafter we use the following shorthand notations: $|e\rangle=\left|F_{e}, m_{e}\right\rangle$ with $m_{e}=-F_{e}, \ldots, F_{e}$, and $|i, m\rangle=\left|F_{i}, m\right\rangle$ with $m=-F_{i}, \ldots, F_{i}(i=1,2)$. The non-Hermitian groundstate effective Hamiltonian has the form

$$
\begin{aligned}
\widehat{H}_{\mathrm{eff}}= & -\frac{\delta_{R}}{2} \sum_{m}(|1, m\rangle\langle 1, m|-| 2, m\rangle\langle 2, m|) \\
& +\widehat{H}_{B}+\langle\widehat{R}\rangle_{v}-i \frac{\Gamma}{2} \widehat{\Pi}_{g} .
\end{aligned}
$$

Here $\delta_{R}=\omega_{1}-\omega_{2}-\Delta_{\mathrm{hfs}}$ is the two-photon (Raman) detuning; $\widehat{H}_{B}$ is the magnetic Hamiltonian, describing the linear Zeeman splitting; the angle brackets $\langle\ldots\rangle_{v}$ denote averaging over atomic velocities with a Maxwell distribution function. The two-photon excitation matrix for an atom moving with a velocity $v$ can be written as

$$
\begin{aligned}
\widehat{R}= & \sum_{i, j, e, m, m^{\prime}}|i, m\rangle \frac{\left.\left.\langle i, m| \widehat{\mathbf{d}} \times \mathbf{E}_{i}\right)^{\dagger}|e\rangle\langle e| \widehat{\mathbf{d}} \times \mathbf{E}_{j}\right)\left|j, m^{\prime}\right\rangle}{\hbar^{2}\left[\left(\delta_{L}-k v-\omega_{e}\right)+i \gamma / 2\right]}\left\langle j, m^{\prime}\right| \\
& +\sum_{i \neq j, e, m, m^{\prime}}|i, m\rangle \frac{\left.\left.\langle i, m| \widehat{\mathbf{d}} \times \mathbf{E}_{j}\right)^{\dagger}|e\rangle\langle e| \widehat{\mathbf{d}} \times \mathbf{E}_{j}\right)\left|i, m^{\prime}\right\rangle}{\hbar^{2}\left[\left(\delta_{L}-k v+\omega_{j}-\omega_{i}-\omega_{e}\right)+i \gamma / 2\right]} \\
& \times\left\langle i, m^{\prime}\right|,
\end{aligned}
$$

where $\widehat{\mathbf{d}}$ is the optical transition dipole moment operator, $\delta_{L}$ is the laser (one photon) detuning, $\gamma$ is the optical linewidth, and $\hbar \omega_{e}$ is the energy of the excited-state hyperfine (HF) levels. Note that $\delta_{L}$ and $\omega_{e}$ are measured from a common zero level (hereafter from the HF level with minimal momentum $F_{e}=I-J_{e}=1$ ). In Eqs. (A2) and (A3) the Doppler shift $k v\left[k=\left(k_{1}+k_{2}\right) / 2\right]$ and strong velocity-changing collisions are taken into account. The matrix $\widehat{R}$ contains resonant (first summation) as well as off-resonant (second summation) contributions to light shifts and optical pumping rates (Hermitian and anti-Hermitian parts, respectively). The nondiagonal $(i \neq j$ ) elements of the resonant term induce Raman coherence between the hyperfine levels of the ground state, which is responsible for the CPT dark resonance.

Here we consider the steady-state regime, setting $(d / d t) \widehat{\sigma}_{g g}=0$ in Eq. (A1). As a spectroscopic signal, we take the total excited-state population $\pi_{e}$, which is proportional to the total light absorption in optically thin media or to the total fluorescence. The following procedure is used to find $\pi_{e}$. From Eq. (A1), the ground-state density matrix $\widehat{\sigma}_{g g}$ is expressed in terms of $\pi_{e}$ and then $\pi_{e}$ is calculated from the normalization condition (A1). In the general case of arbitrary magnetic field this algebraic problem can be solved numerically.

At intermediate magnetic fields, when the magnetically sensitive resonances are well separated from the magnetically insensitive resonances and simultaneously when the two magnetically insensitive resonances $(-1)-(+1)$ and $(+1)-(-1)$ are well overlapped (i.e., when their width is much greater than their Zeeman splitting), the resonance line shape is described with good accuracy by the generalized Lorentzian $[17,18]$

$$
\pi_{e}=C_{0}-\frac{C_{1}(\tilde{\gamma} / 2)^{2}}{(\tilde{\gamma} / 2)^{2}+\left(\delta_{R}-\delta_{0}\right)^{2}}+\frac{C_{2}\left(\delta_{R}-\delta_{0}\right) \tilde{\gamma} / 2}{(\tilde{\gamma} / 2)^{2}+\left(\delta_{R}-\delta_{0}\right)^{2}} .
$$

The coefficients in Eq. (A4) $\left(C_{i}, \tilde{\gamma}\right.$, and $\left.\delta_{0}\right)$ can be analytically expressed in terms of the parameters of the effective groundstate Hamiltonian (A2) in a way similar to the method used in Ref. [17]. Alternatively, they can be extracted from the numerical results by a fitting procedure.
[1] J. Vanier, Appl. Phys. B 81, 421 (2005).

[2] J. Kitching, S. Knappe, N. Vukicevic, L. Hollberg, R. Wynands, and W. Weidmann, IEEE Trans. Instrum. Meas. 49, 1313 (2000).

[3] M. Merimaa, T. Lindwall, I. Tittonen, and E. Ikonen, J. Opt. Soc. Am. B 20, 273 (2003).
[4] Y. Y. Jau, A. B. Post, N. N. Kuzma, A. M. Braun, M. V. Romalis, and W. Happer, Phys. Rev. Lett. 92, 110801 (2004).

[5] A. V. Taichenachev, V. I. Yudin, V. L. Velichansky, S. V. Kargapoltsev, R. Wynands, J. Kitching, and L. Hollberg, JETP Lett. 80, 236 (2004). 
[6] Y.-Y. Jau, E. Miron, A. B. Post, N. N. Kuzma, and W. Happer, Phys. Rev. Lett. 93, 160802 (2004).

[7] T. Zanon, S. Guerandel, E. de Clercq, D. Holleville, N. Dimarcq, and A. Clairon, Phys. Rev. Lett. 94, 193002 (2005).

[8] V. Shah, S. Knappe, L. Hollberg, and J. Kitching, Opt. Lett. 32, 1244 (2007).

[9] N. Vukičević, A. S. Zibrov, L. Hollberg, F. L. Walls, J. Kitching, and H. G. Robinson, IEEE Trans. UFFC 47(5), 1122 (2000).

[10] S. Zibrov, I. Novikova, D. F. Phillips, A. V. Taichenachev, V. I. Yudin, R. L. Walsworth, and A. S. Zibrov, Phys. Rev. A 72, 011801(R) (2005).

[11] I. Novikova, D. F. Phillips, A. S. Zibrov, R. L. Walsworth, A. V. Taichenachev, and V. I. Yudin, Opt. Lett. 31, 622 (2006).

[12] A. V. Taichenachev, V. I. Yudin, V. L. Velichansky, and S. A. Zibrov, JETP Lett. 82, 398 (2005).

[13] G. Kazakov, B. Matisov, I. Mazets, G. Mileti, and J. Delporte, Phys. Rev. A 72, 063408 (2005).

[14] S. A. Zibrov, V. L. Velichansky, A. S. Zibrov, A. V. Taichenachev, and V. I. Yudin, JETP Lett. 82, 477 (2005).

[15] K. Watabe, T. Ilegami, A. Takamizawa, S. Yanagimachi, S. Oshima, and S. Knappe, Appl. Opt. 48, 1098 (2009).
[16] J. S. Guzman, A. Wojciechowski, J. E. Stalnaker, K. Tsigutkin, V. V. Yashchuk, and D. Budker, Phys. Rev. A 74, 053415 (2006).

[17] A. V. Taichenachev, V. I. Yudin, R. Wynands, M. Stahler, J. Kitching, and L. Hollberg, Phys. Rev. A 67, 033810 (2003).

[18] S. Knappe, M. Stahler, C. Affolderbach, A. V. Taichenachev, V. I. Yudin, and R. Wynands, Appl. Phys. B 76, 57 (2003).

[19] D. F. Phillips, I. Novikova, C. Y. T. Wang, M. Crescimanno, and R. L. Walsworth, J. Opt. Soc. Am. B 22, 305 (2005).

[20] J. Vanier, M. W. Levine, D. Janssen, and M. J. Delaney, IEEE Trans. Instrum. Meas. 52, 258 (2003).

[21] J. Vanier, M. W. Levine, D. Janssen, and M. J. Delaney, IEEE Trans. Instrum. Meas. 52, 822 (2003).

[22] E. Breschi, G. Kazakov, R. Lammegger, B. Matisov, L. Windholz, and G. Mileti, IEEE Trans. Ultrason. Ferroelectr. Freq. Control 56, 926 (2009).

[23] E. Breschi, G. Kazakov, R. Lammegger, G. Mileti, B. Matisov, and L. Windholz, Phys. Rev. A 79, 063837 (2009).

[24] E. E. Mikhailov, T. Horrom, N. Belcher, and I. Novikova, J. Am. Opt. Soc. B (to be published).

[25] B. S. Mathur, H. Tang, and W. Happer, Phys. Rev. 171, 11 (1968). 